GRP-057 ERRORS IN MEDICINES PREPARATION AND ADMINISTRATION IN VIETNAMESE HOSPITALS

doi:10.1136/ejhpharm-2013-000276.057

${ }^{1} \mathrm{HT}$ Nguyen, ${ }^{2} \mathrm{TD}$ Nguyen, ${ }^{3} \mathrm{ER}$ van den Heuvel, ${ }^{4} \mathrm{FM}$ Haaijer-Ruskamp, ${ }^{1} \mathrm{~K}$ Taxis. ${ }^{\prime}$ University of Groningen, Pharmacotherapy \& Pharmaceutical Care, Groningen, The Netherlands; 'University of Medicine and Pharmacy at Ho Chi Minh city, Clinical Pharmacy, Ho Chi Minh, Vietnam; 'University Medical Center Groningen, Epidemiology, Groningen, The Netherlands; ${ }^{4}$ University Medical Center Groningen, Clinical Pharmacology, Groningen, The Netherlands

Background Errors in the medication use process are common. Little is known about preparation and administration errors in resource-restricted settings, including Vietnam.

Purpose To determine the frequency, type and severity of medicines preparation errors and administration errors in two Vietnamese hospitals and identify associated factors.

Materials and Methods This is a prospective study using an observation-based approach, carried out in two urban public hospitals. Four trained pharmacy students observed all drugs prepared and administered on six wards, 12 hours per day on 7 consecutive days. Severity of errors was judged by experts using a validated method. Multivariable logistic regression was performed to explore error-associated factors.

Results In total, 2122 out of 5635 preparations or administrations of medicines were erroneous. The error rate was $37.7 \%$ (95\% confidence interval $36.4-38.9 \%$ ). The most frequent errors involved administration technique, preparation technique, omission, and dose $(53.1 \%, 32.6 \%, 5.0 \%$, and $2.6 \%$, respectively). Severity was judged to be moderate in $87.8 \%$ of the cases, followed by severe (8.8\%), and minor errors (3.4\%). Slightly lower medication error rates were observed during afternoon drug rounds than at other times of the day $(32.1 \%$ vs. $39.7 \%, p=0.00)$. Much higher error rates were observed for anti-infective drugs than for any other class of drugs $(77.8 \%$ vs. $28.9 \%$, p $=0.00)$. Medicines with complex preparation procedures were more likely to generate errors than simple ones $(58.1 \%$ vs. $24.7 \%, p=0.00)$, and the error rate of intravenous medicines was much higher than that of other medicines $(73.2 \%$ vs. $12.4 \%, p=0.00)$.

Conclusions This is one of the first large studies investigating medication errors in resource-restricted settings. In around a third of all medicines potentially clinically-relevant errors occurred, which is higher than in most other studies. Administration technique, preparation technique and omission errors were most commonly encountered. Drug round, drug class, complexity of preparation and administration route were error-related factors. Interventions focusing on intravenous medicines with complex preparation procedures are needed to improve patient safety.

No conflict of interest.

\section{GRP-058 ESTABLISHMENT OF A PROGRAMME TO DETECT DRUG INTERACTIONS COMPLEMENTARY TO ELECTRONIC PRESCRIPTIONS}

doi:10.1136/ejhpharm-2013-000276.058

J Bilbao Aguirregomezcorta, C Floristán Imízcoz. Hospital San Eloy, pharmacy, Bilbao, Spain

Background Ours is a 110-bed regional hospital with electronic prescribing throughout. The electronic prescription programme offers allergy alert systems and the need for dosage adjustment in renal and hepatic impairment. However, no warning of potential drug interactions is included.

Purpose To establish a drug interaction screening system to complement the electronic prescribing programme.
Materials and Methods Prospective one-year study (Sept 2011-Sept 2012). Computerized drug interaction alerts can improve the safety, quality and efficiency of care processes and reduce the rate of medicines errors, but sending mass alerts can generate what is known as 'alert fatigue'. For this reason we selected those drugs categorised in Micromedex ${ }^{\circledR} 2.0$ as contraindicated for simultaneous and significant interactions (the interaction can cause death and/or require medical intervention to minimise or avoid serious side effects); drugs classified as D in Lexi-Comp OnlineTM (consider modifying the treatment and X (avoid combination); those classified as clinically important by the Arizona CERT. The interaction was detected by crossing data in Access 2003 with the pharmacotherapeutic profile of patients obtained in the electronic prescribing programme (eOsabide) and a proprietary database which contains a total of 3,133 pairs of interactions.

The report was written in the patient's medical history (Osabide-Global) and acceptance was verified in 24-48 hours.

Results We detected a total of 1996 interactions and 25\% of them were reported, $27 \%$ of which led to changes in medical treatment. The main cause of non-notification (36\%) was that one drug was prescribed if needed.

Conclusions The project was very well accepted among medical professionals and has improved the quality of prescribing. The biggest drawback is the delay in detecting the interaction; it would be helpful if the system generated the warning at the time of prescription.

No conflict of interest.

\section{GRP-059 ETHANOL CONTENT IN CHEMOTHERAPY}

doi:10.1136/ejhpharm-2013-000276.059

M Moreno, A Gil, R Diez, T Molina. Hospital Univesitario de Getafe, Pharmacy, Madrid (Getafe), Spain

Background Ethanol is used as an excipient to enhance the solubility of substances partially soluble in water. For this reason, gemcitabine and paclitaxel, when supplied as an injection concentrate instead of lyophilized powder for reconstitution, contain large amounts of ethanol.

The Spanish Pharmaceutical Association warns that quantities above $3 \mathrm{~g} /$ dose could affect the ability to drive and use machines and interfere with the effects of other drugs.

Chemotherapy Compounding Units pharmacists consider already diluted vials easier to handle and are more willing to use them than vials with lyophilized powder.

Purpose To calculate the average ethanol dose given to oncology patients on gemcitabine and paclitaxel treatment.

Materials and Methods 6-month retrospective study (MarchSeptember 2012) of all patients who had received gemcitabine or paclitaxel.

According to the summary of product characteristics, the ethanol content is:

- Gemcitabine (Actavis ${ }^{\circledR}$ 2,000 mg/50 ml): 9.875 mg ethanol/ mg drug.

- Paclitaxel (Gp-pharm ${ }^{\circledR} 300 \mathrm{mg} / 50 \mathrm{ml}$ ): $65.83 \mathrm{mg}$ ethanol/ mg drug.

Total ethanol dose was then calculated for each patient depending on the chemotherapy dose administered, as shown on clinical records.

Results View table.

Conclusions Ethanol given to these patients may be compared to moderate alcohol consumption. This, together with direct infusion into the blood and the short infusion time, makes it more likely 